\title{
Correlation and Path Coefficient Analysis for Improvement of Seed Yield in Linseed (Linum usitatissimum L.)
}

\author{
Ranjana Patial*, Satish Paul and Devender Sharma \\ Department of Crop Improvement, CSK Himachal Pradesh Krishi Vishvavidyalaya, \\ Palampur-176062, India \\ *Corresponding author
}

\section{A B S T R A C T}

\section{Keywords}

Linseed, Correlation coefficient, Direct effect, Indirect effect and path coefficient analysis

Article Info

Accepted:

16 February 2018 Available Online: 10 March 2018
In this study interrelationship among various morphological and yield related traits was estimated in a set of 34 linseed (Linum usitatissimum L.) genotypes. Genotypic and phenotypic correlation coefficients obtained between different traits was similar in direction, while in magnitude, genotypic correlation higher than the corresponding phenotypic correlations. Correlation studies indicated that seed yield of linseed had significant positive correlation with aerial biomass, harvest index, straw yield, retted straw yield, 1000 seed weight, primary branches per plant, capsules per plant, secondary branches per plant, technical height, fibre yield, plant height, oil content and seeds per capsule. Thus, these thirteen traits can be used as a selection index for improving seed yield. Path coefficient analysis revealed that higher and positive direct effect on seed yield was exhibited by aerial biomass. So, aerial biomass was observed to be best selection parameter because of its direct contribution towards seed yield per plant.

\section{Introduction}

Linseed (Linum usitatissimum L.) commonly known as Alsi, a multipurpose rabi oilseed crop, cultivated for oil and fibre, which belongs to the family Linaceae having 14 genera. Linum has over 200 species with Linum angustifolium Huds $(\mathrm{n}=15)$ being its probable progenitor, native to Mediterranean region and Southwest Asia. Linum usitatissimum is the only economically significant species of the family with semidehiscent and non-dehiscent capsules type (Savita, 2011). It is a self-pollinated crop but cross pollination can take place up to $2 \%$ (Tadesse et al., 2009). Two morphologically distinct cultivated species of linseed are recognized, namely Flax and Linseed. The flax type is commercially grown for the extraction of fibre, whereas the linseed is meant for the extraction of oil from seeds and cake, as a by-product.

Linseed has an important position in Indian economy due to its wide industrial utility. But, the national average productivity of linseed is quite low. Though, it contains about 36 to $48 \%$ oil content which is high in unsaturated fatty acids, especially linolenic acid (Khan et al., 2010). It has drying and hardening properties which is emanated from its high linolenic acid content, thus is mostly used for 
industrial purposes such as manufacturing of paints, varnishes, soaps and printing inks (Wakjira, 2007). The fibre is known for its good quality having high strength and durability, therefore, used in the manufacturing of cloth, water resistant pipes, paper and strawboard. The by-product, oil cake is a valuable dairy feed containing 36 per cent protein, of which 85 per cent is digestible. So, every part of linseed is utilized commercially either directly or after processing with numerous medicinal uses.

Seed yield is a complex character which is dependent on a number of variables. Being a polygenic trait it is greatly influenced by environmental fluctuations. To obtain superior varieties with high yielding potential, the plant breeder have to deal with characters, which are governed by polygenic systems and show continuous variation. Selection at any stage is fruitful only if the breeder is acquainted with the nature and magnitude of variability, association of characters with yield and path coefficient analysis.

The correlation provides the information about the degree but not the cause of association whereas; path coefficient analysis permits a critical examination of various component characters contributing towards the seed yield or any other final product. It measures the relative importance of each factor contributing towards seed yield.

Therefore, knowledge of association among seed yield and its related traits, their relative direct and indirect contribution towards seed yield; is of prime importance in formulating suitable breeding methodology.

Keeping this in view, the present investigation was undertaken with the following objectives: (1) determine phenotypic correlation coefficients among seed yield and yield components and (2) partition the correlation through path coefficient analysis to determine the relative importance of direct and indirect.

\section{Materials and Methods}

An experiment was conducted with 34 genotypes of linseed along with three checks viz., Nagarkot, Him Alsi-2 and Binwa, during rabi crop season 2012-13 at Experimental Farm of the Department of Crop Improvement, CSK HPKV, Palampur. The trial was laid out in Randomized Block Design with three replications having $25 \mathrm{~cm} \times 5 \mathrm{~cm}$ spacing from row to row and plant to plant. The parameters taken at plant basis are primary branches per plant, secondary branches per plant, plant height $(\mathrm{cm})$, technical height $(\mathrm{cm})$, capsules per plant, seeds per capsule, straw yield (g), seed yield per plant $(\mathrm{g})$, retted straw yield $(\mathrm{g})$, fibre yield $(\mathrm{g})$, aerial biomass $(\mathrm{g})$, harvest index $(\%)$. Whereas, days to 50 per cent flowering, days to maturity, 1000-seed weight and oil content (\%) were taken on plot basis.

\section{Statistical analysis}

Phenotypic and genotypic coefficients of correlation were worked out by the procedure of Al- Jibouri et al., (1958) and Dewey and Lu (1959). Because seed yield is the complex outcome of different traits, it was considered as the effect (response) variable or trait, while all other traits were considered as causal (predictor) variables in the cause-and-effect relationship required for path coefficient analysis.

Direct and indirect effects of component characters on grain yield were computed using appropriate correlation coefficient of different component characters as suggested by Wright (1921) and elaborated by Dewey and Lu (1959). The statistical analysis was performed by statistical software WINDOWSTAT 8.0 version. 


\section{Results and Discussion}

\section{Correlation coefficient estimates}

The correlation coefficient is a measure of the degree of association between two traits worked out at the same time. The correlations are important from the point of view of quantitative inheritance of characters and are of practical value for changing two or more traits simultaneously by selection. It resolves the complex relationships between events into simple forms of association. The extent of observed relationship between the characters is known as phenotypic correlation. As such, it does not give the true picture of the genetic relationship between two characters because along with genetic value it includes environmental influence on the covariance between characters. Johnson et al., (1955) stated that estimates of genotypic and phenotypic correlations are useful in planning and evaluating breeding programmes.

Genotypic and phenotypic correlation coefficient was similar in directions, while in magnitude, genotypic correlations were mostly higher than corresponding phenotypic correlations. Similarly, Nagaraja et al., (2009) also reported that genotypic correlation coefficients were higher than their respective phenotypic correlation coefficients for most of the characters. At phenotypic level, seed yield per plant had significant positive associations with primary branches per plant, secondary branches per plant, plant height, technical height, straw yield, retted straw yield, fibre yield, aerial biomass, seeds per capsule, capsules per plant, harvest index and oil content, whereas it showed negative correlation with days to 50 per cent flowering which allows for early flowering (Table 2). Almost similar findings have been reported by most of the workers in linseed viz., Rahimi $e t$ al., (2011), Mohammad et al., (2011), Belete and Yohannes (2013), Tariq et al., (2014) and
Sonwane et al., (2015) and Ibrar et al., (2016). The inter correlation between yield contributing characters may affect the selection for component traits either in favorable or unfavorable direction. Hence, the knowledge on interrelationship between yield component traits may facilitate breeders to decide upon the intensity and direction of selection pressure to be given on related traits for the simultaneous improvement of these traits. Days to 50 per cent flowering had highly significant and positive correlation with days to maturity; primary branches per plant with secondary branches per plant, fibre yield, aerial biomass, capsules per plant and 1000seed weight; secondary branches per plant with straw yield, fibre yield, aerial biomass and capsules per plant; plant height with technical height, straw yield, retted straw yield and aerial biomass; technical height with straw yield, retted straw yield and aerial biomass; straw yield with retted straw yield, fibre yield, aerial biomass and capsules per plant; retted straw yield with fibre yield, aerial biomass and capsules per plant; fibre yield with aerial biomass and capsules per plant; aerial biomass expressed highly significant positive correlation with capsules per plant; seeds per capsule with harvest index; harvest index with 1000-seed weight and oil content and 1000 seed weight had significant positive correlation with oil content.

On the basis of correlation analysis studies, it can be concluded that the selection criteria based on aerial biomass $(\mathrm{r}=0.732 * *)$, harvest index $\left(\mathrm{r}=0.593^{* *}\right)$, straw yield $\left(\mathrm{r}=0.443^{* *}\right)$, retted straw yield $(\mathrm{r}=0.402 * *), 1000$ seed weight $\left(\mathrm{r}=0.378^{* *}\right)$ and primary branches per plant $\left(\mathrm{r}=0.363^{* *}\right)$ can provide better result for improvement of seed yield in linseed. Whereas, secondary branches per plant, plant height, technical height, fibre yield, seeds per capsule, capsules per plant and oil content would also be kept in mind while designing a breeding program. 
Table.1 List of germplasm accessions

\begin{tabular}{|c|c|c|}
\hline S.no. & Genotype & Source/Pedigree \\
\hline 1 & KL-213 & Aoyogi X JRF-2 \\
\hline 2 & KL-216 & Polf-16 X Surbhi \\
\hline 3 & KL-217 & Flak-1X Janaki \\
\hline 4 & KL-218 & RL-50-3 X RL-33-4 \\
\hline 5 & KL-219 & L-1321 X Flak-1 \\
\hline 6 & KL-220 & 89D-2B/4 \\
\hline 7 & KL-221 & $89-2 \mathrm{~B} / 5$ \\
\hline 8 & KL-226 & Aoyogi X JRF-2 \\
\hline 9 & KL-227 & Flak-1 X Janaki \\
\hline 10 & KL-228 & Polf-22 X KL-31 \\
\hline 11 & KL-230 & Aoyogi X RL-33-4 \\
\hline 12 & KL-231 & Polf-16 X KL-1 \\
\hline 13 & KL-232 & Polf-16 X Janki \\
\hline 14 & KL-233 & Flax purple X Gaurav \\
\hline 15 & KL-234 & Polf-22 X Jeevan \\
\hline 16 & KL-236 & Jeevan X Janaki \\
\hline 17 & KL-238 & Aoyogi X Nagarkot \\
\hline 18 & KL-239 & Polf-27 X RL-33-4 \\
\hline 19 & KL-241 & Giza-7 X KLS-1 \\
\hline 20 & KL-242 & Gaurav X KLS-1 \\
\hline 21 & Him Alsi-1 & Palampur \\
\hline 22 & KL-244 & (RLC-29 X Jeevan) X RLC-29 \\
\hline 23 & KL-245 & Jeevan X KLS-1 \\
\hline 24 & KL-246 & Him Alsi-2 X RLC-29 \\
\hline 25 & KL-247 & Neelam X Nagarkot \\
\hline 26 & Jeevan & Palampur \\
\hline 27 & Surbhi & Palampur \\
\hline 28 & Himani & Palampur \\
\hline 29 & Baner & Palampur \\
\hline 30 & Belinka & Exotic collection \\
\hline 31 & Araine & Exotic collection \\
\hline 32 & Nagarkot & Palampur \\
\hline 33 & Him Alsi-2 & Palampur \\
\hline 34 & Binwa & Palampur \\
\hline
\end{tabular}

Checks: Nagarkot, Him Alsi-2 and Binwa 
Table.2 Estimates of correlation coefficients at phenotypic $(\mathrm{P})$ and genotypic $(\mathrm{G})$ levels among different characters of linseed

\begin{tabular}{|c|c|c|c|c|c|c|c|c|c|c|c|c|c|c|c|c|}
\hline Characters & & DTM & PBP & SBP & PH & TH & SY & RSY & FY & $\mathbf{A B}$ & SPC & CPP & HI & SW & OC & $\begin{array}{c}\text { Correlation } \\
\text { with SYP }\end{array}$ \\
\hline \multirow[t]{2}{*}{ DTF } & $\mathbf{P}$ & $0.685^{\text {*** }}$ & -0.145 & -0.057 & 0.052 & 0.117 & -0.186 & -0.111 & $-0.273 * *$ & $-0.243^{*}$ & -0.049 & $-0.311 * *$ & -0.076 & -0.169 & $-0.330 * *$ & $-0.256^{* *}$ \\
\hline & G & $1.018^{* *}$ & $-0.774 * *$ & $-0.311 * *$ & $0.679 * *$ & $0.347^{* *}$ & $-0.250 *$ & $\stackrel{-}{-}$ & $-0.491 * *$ & $\stackrel{-}{-}$ & $0.198^{*}$ & $-0.473 * *$ & -0.013 & $0.299^{-}$ & $-0.782 * *$ & $-0.265 * *$ \\
\hline \multirow[t]{2}{*}{ DTM } & $\mathbf{P}$ & & -0.122 & -0.003 & $0.244 *$ & $0.263^{* * *}$ & 0.070 & 0.004 & $-0.270 * *$ & 0.007 & -0.060 & $-0.315 * *$ & -0.179 & -0.122 & $-0.265^{* *}$ & -0.117 \\
\hline & G & & $-0.984 * *$ & $-0.281 * *$ & $1.233 * *$ & $0.907 * *$ & 0.184 & 0.149 & $-0.648 * *$ & 0.161 & 0.106 & $-0.612 * *$ & -0.099 & $\overline{-}-\overline{268 * *}$ & $-1.007 * *$ & 0.056 \\
\hline \multirow[t]{2}{*}{ PBP } & $\mathbf{P}$ & & & $0.485 * *$ & $-0.209^{*}$ & -0.188 & $0.242^{*}$ & 0.132 & $0.283 * *$ & $0.328 * *$ & 0.017 & $0.487^{* *}$ & 0.164 & $0.341 * *$ & $0.204 *$ & $0.363 * *$ \\
\hline & G & & & $0.632 * *$ & -0.152 & $-0.201 *$ & $0.328^{* * *}$ & $0.276^{* *}$ & $0.434 * *$ & $0.480 * *$ & 0.095 & $0.881^{* * *}$ & $0.335^{* *}$ & $0.474^{* * *}$ & $0.229 *$ & $0.621 * *$ \\
\hline \multirow[t]{2}{*}{$\overline{\text { SBP }}$} & $\mathbf{P}$ & & & & -0.051 & 0.042 & $0.365^{* *}$ & 0.158 & $0.257 * *$ & $0.402 * *$ & 0.055 & $0.441^{* *}$ & 0.002 & 0.096 & 0.002 & $0.315^{* *}$ \\
\hline & G & & & & 0.021 & 0.050 & 0.440 ** & 0.081 & $0.339 * *$ & $0.514 * *$ & 0.053 & $0.722 * *$ & 0.044 & 0.110 & -0.019 & $0.479 * *$ \\
\hline \multirow[t]{2}{*}{ PH } & $\mathbf{P}$ & & & & & $0.842 * *$ & $0.445^{* *}$ & $0.372^{* *}$ & 0.000 & $0.434 * *$ & 0.118 & 0.040 & -0.151 & 0.114 & $-0.234^{*}$ & $0.244^{*}$ \\
\hline & G & & & & & 1.010 ** & $0.574 * *$ & $0.795^{* *}$ & -0.069 & 0.576 ** & 0.113 & $-0.209 *$ & -0.182 & 0.139 & $-0.323 * *$ & $0.373 * *$ \\
\hline \multirow[t]{2}{*}{$\overline{\mathrm{TH}}$} & $\mathbf{P}$ & & & & & & $0.418^{* *}$ & $0.470 * *$ & -0.069 & $0.427 * *$ & 0.076 & -0.050 & -0.104 & $0.212 *$ & -0.138 & $0.277 * *$ \\
\hline & G & & & & & & $0.469^{* *}$ & $0.658^{* *}$ & -0.116 & $0.497 * *$ & 0.097 & -0.180 & -0.078 & $0.203 *$ & -0.159 & $0.375^{* *}$ \\
\hline \multirow[t]{2}{*}{$\overline{\text { SY }}$} & $\mathbf{P}$ & & & & & & & $0.623 * *$ & $0.509 * *$ & $0.935 * *$ & -0.125 & $0.369 * *$ & $-0.443 * *$ & 0.053 & $-0.245^{*}$ & $0.443 * *$ \\
\hline & G & & & & & & & $0.834 * *$ & $0.568 * *$ & $0.947 * *$ & -0.194 & $0.515^{* *}$ & $-0.475^{* *}$ & 0.023 & $-0.269 * *$ & 0.496 ** \\
\hline \multirow[t]{2}{*}{$\overline{\mathbf{R S Y}}$} & $\mathbf{P}$ & & & & & & & & $0.297 * *$ & $0.632 * *$ & 0.017 & $0.336^{* * *}$ & -0.128 & $0.239 *$ & -0.186 & $0.402 * *$ \\
\hline & G & & & & & & & & $0.368^{* *}$ & $0.816^{* *}$ & -0.011 & $0.408^{* *}$ & $-0.311^{* *}$ & $0.281^{* *}$ & $-0.220^{*}$ & $0.484 * *$ \\
\hline \multirow[t]{2}{*}{ FY } & $\mathbf{P}$ & & & & & & & & & $0.488^{* *}$ & $-0.224 *$ & $0.514 * *$ & $-0.201^{*}$ & -0.076 & -0.020 & $0.256^{* *}$ \\
\hline & G & & & & & & & & & $0.545^{* *}$ & $-\overline{-}$ & $0.748 * *$ & $-0.268 * *$ & -0.107 & -0.045 & $0.300 * *$ \\
\hline \multirow[t]{2}{*}{$\mathbf{A B}$} & $\mathbf{P}$ & & & & & & & & & & -0.013 & $0.409^{* *}$ & -0.102 & 0.190 & -0.092 & $0.732 * *$ \\
\hline & G & & & & & & & & & & -0.075 & $0.543^{* *}$ & -0.168 & 0.175 & -0.084 & $0.748^{* *}$ \\
\hline \multirow[t]{2}{*}{ SPC } & $\mathbf{P}$ & & & & & & & & & & & 0.090 & $0.327^{* *}$ & 0.063 & 0.086 & $0.206^{*}$ \\
\hline & G & & & & & & & & & & & 0.109 & $0.423 * *$ & 0.048 & 0.141 & $0.198^{*}$ \\
\hline \multirow[t]{2}{*}{ CPP } & $\mathbf{P}$ & & & & & & & & & & & & 0.023 & 0.011 & -0.022 & $0.325^{* *}$ \\
\hline & G & & & & & & & & & & & & -0.094 & 0.006 & 0.016 & $0.405^{* *}$ \\
\hline \multirow[t]{2}{*}{ HI } & $\mathbf{P}$ & & & & & & & & & & & & & $0.324 * *$ & $0.430 * *$ & $0.593 * *$ \\
\hline & G & & & & & & & & & & & & & $0.434 * *$ & $0.612^{* *}$ & $0.528 * *$ \\
\hline \multirow[t]{2}{*}{ SW } & $\mathbf{P}$ & & & & & & & & & & & & & & $0.316^{* * *}$ & $0.378 * *$ \\
\hline & G & & & & & & & & & & & & & & $0.363^{* *}$ & $0.427 * *$ \\
\hline \multirow[t]{2}{*}{$\overline{\mathrm{OC}}$} & $\mathbf{P}$ & & & & & & & & & & & & & & & $0.238^{*}$ \\
\hline & G & & & & & & & & & & & & & & & $0.328 * *$ \\
\hline
\end{tabular}

*Significant at 5 per cent level; **Significant at 1 per cent level

DTF- Days to 50\% flowering; DTM- Days to maturity; PB- Primary branches per plant; SB- Secondary branches per plant; PH-Plant height (cm); TH- Technical height (cm); SY- Straw yield (g); RSY- Retted straw yield (g); FY- Fibre yield (g); AB- Aerial biomass (g); SPC-Seeds per capsule; CPP-Capsules per plant; HIHarvest index (\%); SW-1000 Seed weight; OC- Oil content (\%); SYP-Seed yield per plant. 
Table.3 Estimates of direct and indirect phenotypic and genotypic effects of different characters on seed yield

\begin{tabular}{|c|c|c|c|c|c|c|c|c|c|c|c|c|c|c|c|c|c|}
\hline Traits & & DTF & DTM & PBP & SBP & PH & TH & SY & RSY & FY & AB & SPC & CPP & HI & TSW & $\mathrm{OC}$ & $\begin{array}{l}\text { Correlation } \\
\text { with seed } \\
\text { yield }\end{array}$ \\
\hline \multirow[t]{2}{*}{ DTF } & $\mathbf{P}$ & 0.0028 & 0.0019 & -0.0004 & -0.0002 & 0.0001 & 0.0003 & -0.0005 & -0.0003 & -0.0008 & -0.0007 & -0.0001 & -0.0009 & -0.0002 & -0.0005 & -0.0009 & $-0.256^{* *}$ \\
\hline & G & 0.0030 & 0.0031 & -0.0023 & -0.0009 & 0.0020 & 0.0010 & -0.0007 & -0.0011 & -0.0015 & -0.0009 & 0.0006 & -0.0014 & 0.0000 & -0.0009 & -0.0023 & $-0.265 * *$ \\
\hline \multirow[t]{2}{*}{$\overline{D T M}$} & $\mathbf{P}$ & -0.0019 & -0.0028 & 0.0003 & 0.0000 & -0.0007 & -0.0007 & -0.0002 & 0.0000 & 0.0008 & 0.0000 & 0.0002 & 0.0009 & 0.0005 & 0.0003 & 0.0007 & -0.117 \\
\hline & G & -0.0074 & -0.0073 & 0.0072 & 0.0021 & -0.0090 & -0.0066 & -0.0013 & -0.0011 & 0.0047 & -0.0012 & -0.0008 & 0.0045 & 0.0007 & 0.0020 & 0.0073 & 0.056 \\
\hline \multirow[t]{2}{*}{ PBP } & $\mathbf{P}$ & 0.0002 & 0.0002 & -0.0015 & -0.0007 & 0.0003 & 0.0003 & -0.0004 & -0.0002 & -0.0004 & -0.0005 & 0.0000 & -0.0007 & -0.0002 & -0.0005 & -0.0003 & $0.363 * *$ \\
\hline & G & -0.0117 & -0.0149 & 0.0151 & 0.0095 & -0.0023 & -0.0030 & 0.0050 & 0.0042 & 0.0065 & 0.0072 & 0.0014 & 0.0133 & 0.0051 & 0.0072 & 0.0035 & $0.621 * *$ \\
\hline \multirow[t]{2}{*}{ SBP } & $\mathbf{P}$ & -0.0001 & 0.0000 & 0.0010 & 0.0020 & -0.0001 & 0.0001 & 0.0007 & 0.0003 & 0.0005 & 0.0008 & 0.0001 & 0.0009 & 0.0000 & 0.0002 & 0.0000 & $0.315 * *$ \\
\hline & G & -0.0010 & -0.0009 & 0.0020 & 0.0031 & 0.0001 & 0.0002 & 0.0014 & 0.0003 & 0.0011 & 0.0016 & 0.0002 & 0.0022 & 0.0001 & 0.0003 & -0.0001 & $0.479 * *$ \\
\hline \multirow[t]{2}{*}{ PH } & $\mathbf{P}$ & 0.0003 & -0.0014 & -0.0012 & -0.0003 & 0.0057 & 0.0048 & 0.0025 & 0.0021 & 0.0000 & 0.0025 & 0.0007 & 0.0002 & -0.0009 & 0.0006 & -0.0013 & $0.244 * *$ \\
\hline & G & 0.0155 & 0.0281 & -0.0035 & 0.0005 & 0.0228 & 0.0230 & 0.0131 & 0.0181 & -0.0016 & 0.0131 & 0.0026 & -0.0048 & -0.0041 & 0.0032 & -0.0074 & $0.373 * *$ \\
\hline \multirow[t]{2}{*}{ TH } & $\mathbf{P}$ & -0.0005 & -0.0012 & 0.0009 & -0.0002 & -0.0039 & -0.0046 & -0.0019 & -0.0022 & 0.0003 & -0.0020 & -0.0003 & 0.0002 & 0.0005 & -0.0010 & 0.0006 & $0.277 * *$ \\
\hline & G & -0.0054 & -0.0142 & 0.0032 & -0.0008 & -0.0159 & -0.0157 & -0.0074 & -0.0103 & 0.0018 & -0.0078 & -0.0015 & 0.0028 & 0.0012 & -0.0032 & 0.0025 & $0.375 * *$ \\
\hline \multirow[t]{2}{*}{ SY } & $\mathbf{P}$ & 0.3562 & -0.1340 & -0.4634 & -0.6989 & -0.8521 & -0.8004 & -1.1948 & -1.1929 & 0.9746 & -1.7903 & 0.2394 & -0.7066 & 0.8483 & -0.1015 & 0.4691 & $0.443 * *$ \\
\hline & G & 0.5893 & -0.4341 & -0.7745 & -1.0389 & -1.3539 & -1.1071 & -2.3591 & -1.9677 & -1.3397 & -2.2343 & 0.4577 & -1.2147 & 1.1215 & -0.0531 & 0.6334 & $0.493 * *$ \\
\hline \multirow[t]{2}{*}{ RSY } & $\mathbf{P}$ & -0.0002 & 0.0000 & 0.0003 & 0.0003 & 0.0007 & 0.0009 & 0.0012 & 0.0020 & 0.0006 & 0.0013 & 0.0000 & 0.0007 & -0.0003 & 0.0005 & -0.0004 & $0.402 * *$ \\
\hline & G & -0.0006 & 0.0002 & 0.0004 & 0.0001 & 0.0013 & 0.0011 & 0.0013 & 0.0016 & 0.0006 & 0.0013 & 0.0000 & 0.0007 & -0.0005 & 0.0004 & -0.0004 & $0.484 * *$ \\
\hline \multirow[t]{2}{*}{ FY } & $\mathbf{P}$ & 0.0001 & 0.00001 & -0.0001 & -0.0001 & 0.0000 & 0.0000 & -0.0002 & -0.0001 & -0.0003 & -0.0001 & 0.0001 & -0.0002 & 0.0001 & 0.0000 & 0.0000 & $0.256^{* *}$ \\
\hline & G & -0.0005 & -0.0007 & 0.0005 & 0.0004 & -0.0001 & -0.0001 & 0.0006 & 0.0004 & 0.0011 & 0.0006 & -0.0003 & 0.0008 & -0.0003 & -0.0001 & 0.0000 & $0.300 * *$ \\
\hline \multirow[t]{2}{*}{$\mathbf{A B}$} & $\mathbf{P}$ & -0.6129 & 0.0177 & 0.8272 & 1.0139 & 1.0946 & 1.0769 & 2.3582 & 1.5940 & 1.2308 & 2.5221 & -0.0328 & 1.0315 & -0.2573 & 0.4792 & -0.2320 & $0.732 * *$ \\
\hline & G & -0.8557 & 0.4774 & 1.4220 & 1.5209 & 1.7069 & 1.4717 & 2.8051 & 2.4165 & 1.6136 & 2.9618 & -0.2227 & 1.6080 & -0.4988 & 0.5177 & -0.2482 & $0.748 * *$ \\
\hline \multirow[t]{2}{*}{ SPC } & $\mathbf{P}$ & 0.0001 & 0.0001 & 0.0000 & -0.0001 & -0.0002 & -0.0001 & -0.0002 & 0.0000 & 0.0004 & 0.0000 & -0.0016 & -0.0001 & -0.0005 & -0.0001 & -0.0004 & $0.206^{*}$ \\
\hline & G & 0.0010 & 0.0005 & 0.0005 & 0.0003 & 0.0006 & 0.0005 & -0.0010 & -0.0001 & -0.0014 & -0.0004 & 0.0052 & 0.0006 & 0.0022 & 0.0002 & 0.0007 & $0.196^{*}$ \\
\hline \multirow[t]{2}{*}{ CPP } & $\mathbf{P}$ & 0.0002 & 0.0002 & -0.0003 & -0.0003 & 0.0000 & 0.0000 & -0.0002 & -0.0002 & -0.0003 & -0.0002 & -0.0001 & -0.0006 & 0.0000 & 0.0000 & 0.0000 & $0.325^{* *}$ \\
\hline & G & 0.0080 & 0.0103 & -0.0149 & -0.0122 & 0.0035 & 0.0030 & -0.0087 & -0.0069 & -0.0126 & -0.0092 & -0.0018 & -0.0169 & 0.0016 & -0.0001 & -0.0003 & $0.405 * *$ \\
\hline \multirow[t]{2}{*}{ HI } & $\mathbf{P}$ & -0.0002 & -0.0005 & 0.0004 & 0.0000 & -0.0004 & -0.0003 & -0.0012 & -0.0003 & -0.0005 & -0.0003 & 0.0009 & 0.0001 & 0.0027 & 0.0009 & 0.0012 & $0.593 * *$ \\
\hline & G & 0.0013 & 0.0100 & -0.0339 & -0.0045 & 0.0184 & 0.0079 & 0.0482 & 0.0315 & 0.0272 & 0.0171 & -0.0428 & 0.0096 & -0.1013 & -0.0440 & -0.0620 & $0.528 * *$ \\
\hline \multirow[t]{2}{*}{ TSW } & $\mathbf{P}$ & 0.0002 & 0.0002 & -0.0004 & -0.0001 & -0.0001 & -0.0003 & -0.0001 & -0.0003 & 0.0001 & -0.0002 & -0.0001 & 0.0000 & -0.0004 & -0.0013 & -0.0004 & $0.378 * *$ \\
\hline & G & 0.0013 & 0.0011 & -0.0020 & -0.0005 & -0.0006 & -0.0009 & -0.0001 & -0.0012 & 0.0005 & -0.0007 & -0.0002 & 0.0000 & -0.0018 & -0.0042 & -0.0015 & $0.427 * *$ \\
\hline \multirow[t]{2}{*}{ OC } & $\mathbf{P}$ & -0.0006 & -0.0005 & 0.0003 & 0.0000 & -0.0004 & -0.0002 & -0.0004 & -0.0003 & 0.0000 & -0.0002 & 0.0001 & 0.0000 & 0.0007 & 0.0005 & 0.0017 & $0.238^{* *}$ \\
\hline & G & -0.0024 & -0.0031 & 0.0007 & -0.0001 & -0.0010 & -0.0005 & -0.0008 & -0.0007 & -0.0001 & -0.0003 & 0.0004 & 0.0000 & 0.0019 & 0.0011 & 0.0031 & $0.328 * *$ \\
\hline
\end{tabular}

Residual effects $(\mathrm{P})=0.018 ;(\mathrm{G})=0.009$; Bold values indicates direct effects; *Significant at 5 per cent level; **Significant at 1 per cent level 


\section{Estimates of direct and indirect effects}

In order to understand the causal factors of correlations among the characters studied, the estimates of direct and indirect contribution of different characters towards seed yield per plant, the path coefficient analysis was done (Table 3). The direct and indirect effects of genotypic path coefficient were higher in magnitude than the corresponding phenotypic path coefficients. Similar finding with respect to path coefficients have been reported by Gauraha and Rao (2011) and Reddy et al., (2013). Although 13 traits viz., aerial biomass, harvest index, straw yield, retted straw yield, 1000-seed weight, primary branches per plant, capsules per plant, secondary branches per plant, technical height, fibre yield, plant height, oil content and seeds per capsule showed positive correlation with the seed yield per plant and one trait viz., days to 50 per cent flowering showed negative correlation. However, the direct and indirect contribution of correlation revealed the positive direct effect for aerial biomass only, which is nullified by straw yield. So, for the direct selection we can go for aerial biomass only in order to improve seed yield.

On partitioning the components for correlation of seed yield with characters showing positive correlation, direct effect were found to be low indicating that while selecting these characters seed yield per plant can't be improved through these characters. Their indirect effects through aerial biomass were high, therefore harvest index, straw yield, retted straw yield, 1000 seed weight, primary branches per plant, capsules per plant, secondary branches per plant, technical height, fibre yield, plant height, oil content and seeds per capsule contributed indirectly through aerial biomass. Similar results were observed by Tadesse et al., (2009) for harvest index and aerial biomass; Bindra (2012) observed that aerial biomass was the main determinant of seed yield per plant and Paul et al., (2015) also found biological yield/plot had the greatest positive direct effect on seed yield/plot in both the seasons. The results of the present study suggest that for improving yield selection should be made for aerial biomass. Hence, based upon correlation and path coefficient analysis, aerial biomass was observed to be best selection parameter because of its direct contribution towards seed yield per plant.

\section{References}

Al-Jibouri, H. A., Millar, P. A. and Robinson, H. P. 1958. Genotypic and environmental variance and covariances in an upland cotton cross of interspecific origin. Agron. J. 50: 633637.

Belete, Y. S. and Yohannes, M. T. W. 2013. Genetic variation of different crosses of linseed genotypes for some agromorphological traits. Asian J. crop Sci. 5: 436-443.

Bindra, S. 2012. Genetic diversity and association studies in linseed (Linum usitatissimum L.). M.Sc thesis, p 72. Department of crop improvement, CSK Himachal Pradesh Krishi Vishvavidyalaya, Palampur, India.

Dewey, D. R. and Lu, K.H. 1959. A correlation and path coefficient analysis of components of crested wheat grass and seed production. Agron. J. 51: 515518.

Gauraha, D. and Rao, S.S. 2011. Association Analysis for Yield and its Characters in Linseed (Linum usitatissimum L.). Res. J. Agric. Sci. 2: 258-260.

Ibrar, D., Ahmad, R., Mirza, M.Y., Mahmood, T., Khan, M. A. and Iqbal, M.S. 2016. Correlation and Path analysis for yield and yield components 
in Linseed (Linum usitatissimum L.). J. Agric. Res. 54:153-159.

Johnson, H.W., Robinson, H. F. and Comstock, R.E. 1955. Estimates of genetic and environmental variability in soybean. Agron. J. 47: 314-318.

Khan, M.L., Sharif, M. and Sarwa, M. 2010. Chemical Composition of Different Varieties of Linseed. Pak. Vet. J. 02538318.

Mohammad, M. R., Mohammad, A. Z. and Ali, A. 2011. Selection criteria of flax (Linum usitatissimum L.) for seed yield, yield components and biochemical compositions under various planting dates and nitrogen. Afr. J. Agric. Res. 6: 3167-3175.

Nagaraja, T. E., Ajit, K. R. and Golasangi, B. S. 2009. Genetic variability, correlation and path analysis in linseed. $J$. Maharashtra Agric. Uni. 34: 282-285.

Paul, S., Bhateria, S. and kumara, A.2015. Genetic variability and interrelationships of seed yield and yield components in Linseed (Linum usitatissimum L.). SABRAO J. Breed Genet. 47: 375-383.

Rahimi, M. M., Zarei, A. M. and Arminian, A. 2011. Selection criteria of flax (Linum usitatissimum L.) for seed yield, yield components and biochemical composition under various planting dates and nitrogen. African J. Agric. Res.6: 3167-3175.

Reddy, M. P., Reddy, R. B., Arsul, B.T. and Maheshwari, J. J.2013. Character association and Path Coefficient Studies in Linseed. Int. J. Curr. Microbiol. Appl Sci. 2: 250-254.

Sonwane, A. G., Kathale, M. N., Ghodke, M. K. and Ingle, A. U. 2015. Correlation and Path Analysis Studies for Yield and Yield Contributing Characters in Linseed (Linum usitatissimum). Trends Biosciences 8(14):3655-3659.

Tadesse, T., Singh, H. and Weyessa, B. 2009. Correlation and path coefficient analysis among seed yield traits and oil content in Ethiopian linseed Germplasm. Int. J. Sustain. Crop Prod. 4: 08-16.

Tariq, A. M., Hussain, T., Ahmad, I., Saghir, M., Batool, M., Safdar, M. and Tariq, M. 2014. Association analysis in Linseed (Linum Usitatissimum L.). J. Biol. Agric. Healthc. 4: 6.

Wakjira, A. 2007. Linseed (Linum usitatissimum L.) In: Vegetable oils and fats, Plant Resources of Tropical Africa (PROTA), Vandervosson, H.AM. and G.S.M Kamilo (Eds.). PROTA Foundation, Wageningen, Netherland. P. 108-115.

Wright, S. 1921. Correlation and causation. $J$. Agric. Res.20: 557-585.

\section{How to cite this article:}

Ranjana Patial, Satish Paul and Devender Sharma. 2018. Correlation and Path Coefficient Analysis for Improvement of Seed Yield in Linseed (Linum usitatissimum L.). Int.J.Curr.Microbiol.App.Sci. 7(03): 1853-1860. doi: https://doi.org/10.20546/ijcmas.2018.703.219 